\title{
Micromechanical Analyses of Debonding and Matrix Cracking in Dual-Phase Materials
}

\author{
Legarth, Brian Nyvang; Yang, Qingda
}

Published in:

Journal of Applied Mechanics

Link to article, DOI:

$10.1115 / 1.4032690$

Publication date:

2016

Document Version

Peer reviewed version

Link back to DTU Orbit

Citation (APA):

Legarth, B. N., \& Yang, Q. (2016). Micromechanical Analyses of Debonding and Matrix Cracking in Dual-Phase Materials. Journal of Applied Mechanics, 83(5), 051006. https://doi.org/10.1115/1.4032690

\section{General rights}

Copyright and moral rights for the publications made accessible in the public portal are retained by the authors and/or other copyright owners and it is a condition of accessing publications that users recognise and abide by the legal requirements associated with these rights.

- Users may download and print one copy of any publication from the public portal for the purpose of private study or research.

- You may not further distribute the material or use it for any profit-making activity or commercial gain

- You may freely distribute the URL identifying the publication in the public portal

If you believe that this document breaches copyright please contact us providing details, and we will remove access to the work immediately and investigate your claim. 


\title{
Micro-mechanical analyses of debonding and matrix cracking in dual-phase materials
}

\author{
Brian Nyvang Legarth \\ Associate professor \\ Department of Mechanical Engineering, Solid Mechanics \\ Technical university of Denmark \\ DK-2800 Kgs. Lyngby, Denmark \\ Email: bnl@mek.dtu.dk \\ Qingda Yang \\ Associate professor \\ Department of Mechanical and Aerospace Engineering \\ University of Miami, Coral Gables \\ FL 33124, USA \\ Email: qdyang@miami.edu
}

Failure in elastic dual-phase materials under transverse tension is studied numerically. Cohesive zones represent failure along the interface and the augmented FEM is used for matrix cracking. Matrix cracks are formed at an angle of $55^{\circ}-60^{\circ}$ relatively to the loading direction, which is in good agreement with experiments. Matrix cracks initiate at the tip of the debond, and for equi-biaxial loading cracks are formed at both tips. For elliptical reinforcement the matrix cracks initiate at the narrow end of the ellipse. The load carrying capacity is highest for ligaments in the loading direction greater than that of the transverse direction.

$\begin{array}{ll}\text { Nomenclature } \\ \overline{\mathbf{f}}_{\alpha}^{\prime}, \mathbf{f}_{\alpha}^{\prime}, \overline{\mathbf{F}}_{\alpha}^{\prime}, \mathbf{F}_{\alpha}^{\prime} & \text { Local and global load vectors } \\ \mathbf{k}_{\alpha}^{\prime}, \mathbf{K} & \text { Local and global stiffness matrices } \\ \mathbf{N}_{\alpha}, \mathbf{N}_{\alpha}^{\prime} & \text { Standard and transformed shape functions } \\ \mathbf{u}_{\alpha}^{\prime}, \mathbf{d}_{\alpha}^{\prime}, \mathbf{d} & \text { Displacements in FEM } \\ a_{\mathrm{i}}, b_{\mathrm{i}}, a_{\mathrm{c}}, & \text { Parameters describing the geometry } \\ b_{\mathrm{c}}, \beta_{\mathrm{i}}, \Omega, S & \text { Young's modulus, Poisson's ratio } \\ E_{m}, v_{m} & \text { Volume fraction of reinforcement } \\ f & \text { Overall average deformation gradients } \\ F_{\alpha \beta}^{\mathrm{AV}} & \text { Surface tractions } \\ T_{i} & \text { Current normal and tangential tractions at } \\ T_{\mathrm{n}}, T_{\mathrm{t}} & \text { cohesive interface } \\ u_{\mathrm{n}}, u_{\mathrm{t}} & \text { Current normal and tangential separations at } \\ u_{\alpha}, \Delta_{1} & \text { cohesive interface } \\ x_{i} & \text { Displacements, prescribed displacement } \\ \alpha, \beta, \gamma & \text { Cartesian reference coordinate system } \\ \Gamma, \mathcal{G} & \text { Indices } \\ & \text { Traction-separation work }\end{array}$

$\begin{array}{ll}\delta_{\mathrm{n} 1}, \delta_{\mathrm{t} 1}, \delta_{\mathrm{n}}^{c}, \delta_{\mathrm{t}}^{c} & \text { Input parameters for cohesive interface } \\ \kappa & \text { Load parameter } \\ \lambda & \text { Non-dimensional interfacial separation } \\ \sigma_{\max }, \tau_{\max } & \text { Maximum normal and shear stress across the } \\ \sigma_{1}, S_{\alpha \beta}, \varepsilon_{1} & \text { cohesive interface } \\ \sigma_{\alpha \beta}, \varepsilon_{\alpha \beta} & \text { Overall average stresses and strain } \\ \end{array}$

\section{Introduction}

Heterogeneous dual-phase materials with a solid second-phase inclusion embedded in a matrix material are often observed. The presence of the second-phase may be intentional reinforcement as in aligned fiber composites or unintentional impurities in a "pure" material, for instance oxides in aluminum [1]. Typically, a high volume fraction of the reinforcement is seen for composites, whereas the impurities appear at a lower volume fraction. Composite materials form a class of materials that has highly attractive mechanical properties, i.e. high specific stiffness, good damping and creep capabilities and high wear resistance. However, independent of the volume fraction, the reinforcement acts as a local stress intensifier, and macroscopic failure often initiates from the second-phase inclusion leading to reduced ductility and poor fracture properties $[2,3]$.

Detailed micro-mechanical studies have been widely used to analyze the governing failure mechanisms. In doing so, each reinforcing inclusion with its mechanical properties, bonding capabilities, defects, size and position can be accounted for [4]. For aligned fiber composites different failure mechanisms have been identified depending on the loading conditions and the properties of the constituents. Compres- 
sion leads to micro buckling of the fibers and the formation of shear bands [5]. In longitudinal tension fiber pull-out or fiber breakage can occur depending on the bonding between fiber and matrix. In transverse tension a weak interface will cause a debonding crack to evolve, which upon further loading may propagate into the matrix [6]. The latter failure mechanism is also very critical for the case of impurities with low volume fractions. Hence, the aim of the present paper is to study this generic failure mechanism, where debonding initiates at the interface of the second-phase inclusion, but eventually arrests and kinks into the surrounding material.

Recently, Vajari et al. have conducted a micromechanical cell study with multiple, randomly distributed reinforcement [7]. The effects of porosities on the macroscopic failure locus was investigated and compared to the criterion of Puck [8]. They used a simple damage model to represent matrix cracking and they found a significant influence of the porosities, especially in compression. From a computational point of view, the computational cost grows rapidly the more features the model contains. Hence, often a relatively small amount of reinforcing inclusions is taken into account or simpler material models are adopted. The author has studied the effect of plastic anisotropy on interfacial debonding using a single fiber cell, where the possibilities of matrix cracking was excluded [9]. However, the strain field in the matrix clearly indicates, that the interface crack arrests and tries to kink into the matrix for further propagation. The aim of this work is to extend this previous work in Ref. [9] in order to study debonding cracks that kink into the matrix.

In the present study both single and multiple inclusion cells are adopted to study both interface debonding and matrix cracking. The main focus will be on the single inclusion cell. However, it is an inherent feature of such single inclusion cells, that the strain field near all neighboring inclusions are identical to the one analyzed, resulting in a similar failure behavior. In reality damage initiates at the weakest point causing the stresses locally to be redistributed. Hence, a new material point some distance away will eventually experience the stress being sufficiently high to cause failure. For ceramic-matrix composites explicit expressions for the distance needed in order to gain full load carrying capacity again can be found [10]. Here, a multiple inclusion cell is therefore also used to investigate the effect of neighboring inclusions that debonds at a larger load. The novelty of this work is also to incorporate geometrical anisotropy to identify its effect on matrix cracking as well as the overall load carrying capacity when a debonding crack kinks into matrix. Interface debonding and matrix cracking are taking into account by cohesive zone modeling and the augmented FEM technique (A-FEM), respectively. Cohesive zone modeling has been widely used, not only for composite materials, but also for fracture problems in homogeneous materials [11]. The A-FEM technique is developed by Yang and co-workers [12], and is a general method to handle discontinuities in the domain of interest. An alternative procedure is the extended FEM (X-FEM) developed by Belytschko and co-workers [13]. The main difference is, that A-FEM adopts standard shape functions, whereas X-FEM uses an enriched set of shape functions. Also, a numerical scheme, where cohesive elements are inserted adaptively to represent dynamic fracture in brittle materials has been developed [14].

In the present paper focus will be on the overall, homogenized stress-strain response as well as the initiation and path of the matrix cracks.

\section{Problem Formulation}

An elastic matrix material is reinforced by a second phase of inclusions, which are significantly stiffer than the surrounding matrix material. Thus, the reinforcements can be regarded as rigid. It is noted, that this is just a simplification for convenience and it is not an inherent part of the methodology used. General studies of such dual-phase materials require full three dimensional analyses in order to describe the geometry of the reinforcements as well as general loading conditions. However, as progressive interface debonding as well a matrix cracking are considered in this work, such analyses are complicated and time consuming. Upon simplification, the original problem is therefore often considered as plane with in-plane loading. This leads to less realistic reinforcement geometry, but it is expected to give a good understanding of the interfacial debonding as well as matrix cracking. At this point there are two possibilities: A plane strain or a plane stress model. If the dimension of the matrix material in the out of plane direction is small and comparable to the out of plane dimension of the inclusions, as can be the case in a thin sheet, plane stress state near the inclusions is an acceptable assumption [9]. On the other hand, if the out of plane dimension is much longer than the cell dimensions, the material will behave like a bulk material constraining the out of plane strain, and the plane strain cell model is a valid approximation. Here, the plane strain assumption is chosen. Thus, the out of plane dimension is assumed large enough to constrain the out of plane deformations.

Fig. 1(a) shows the material with idealized periodically arranged rigid reinforcements and Fig. 1(b) defines the biaxially loaded unit cell adopted for most of the micromechanical investigations of failure by debonding and matrix cracking conducted here. However, results using a cell similar to Fig. 1(a) is also presented. A fixed Cartesian coordinate system, $x_{i}$, is aligned with the cell edges and located at the center of the reinforcement. The reinforcement is assumed elliptic with the dimensions $\left(2 a_{\mathrm{i}}, 2 b_{\mathrm{i}}\right)$, and the orientation relatively to the $x_{1}$-direction is given by $\beta_{\mathrm{i}}$. The volume fraction of the reinforcing inclusion is $f=\pi a_{\mathrm{i}} b_{\mathrm{i}} /\left(4 a_{\mathrm{c}} b_{\mathrm{c}}\right)$, as the dimensions of the cell are $2 a_{\mathrm{c}}$ and $2 b_{\mathrm{c}}$, see Fig. 1(b). Due to progressive matrix cracking and the orientation of the elliptical reinforcement shear stresses can develop at the cell sides resulting in some wavy deformations of the initially straight edges. In order to allow for such deformations periodical boundary conditions are prescribed. The basic idea is to ensure compatibility as well as force equilibrium across 


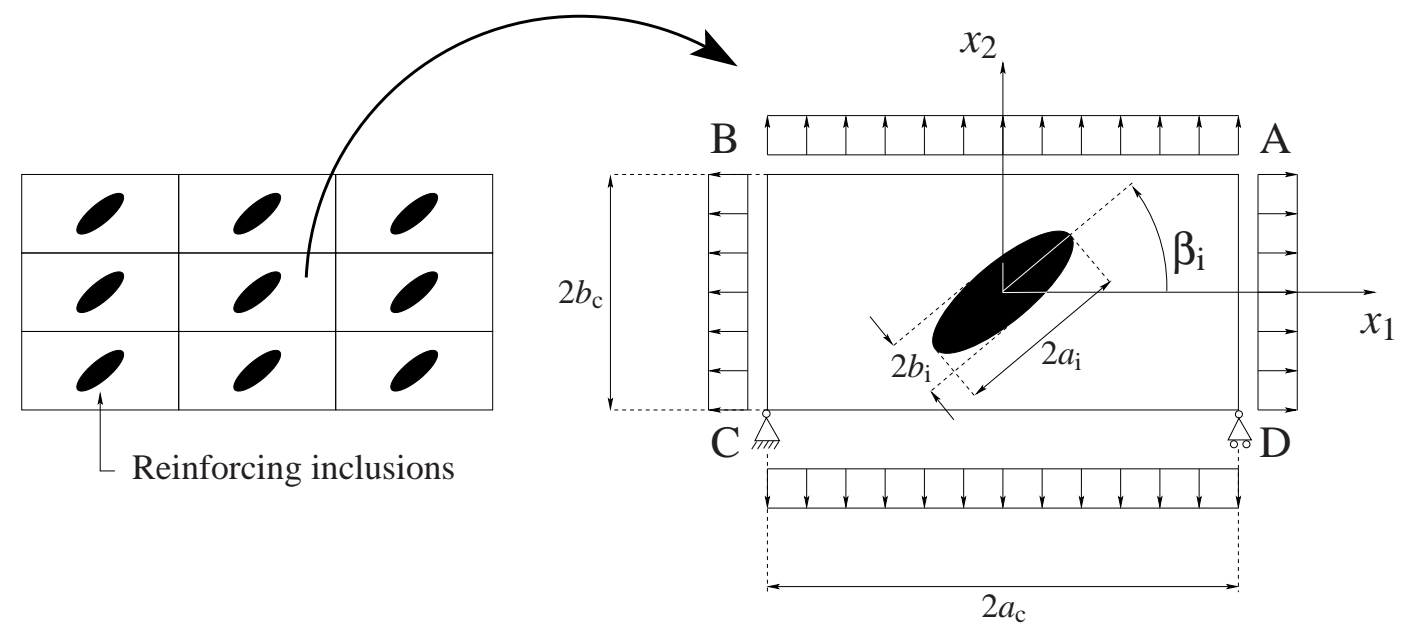

(a)

(b)

Fig. 1. The plane strain cell model for rigid elliptical reinforcing inclusions. (a) Assumed periodically arranged reinforcement in the overall heterogeneous material. (b) The cell used for modeling is shown with initial dimensions, loads, supports and coordinate system.

any cell edge. The compatibility conditions are [15]

$$
\begin{aligned}
& u_{1}\left(x_{1},-b_{\mathrm{c}}\right)=u_{1}\left(x_{1}, b_{\mathrm{c}}\right)-u_{1}^{\mathrm{B}} \\
& u_{2}\left(x_{1},-b_{\mathrm{c}}\right)=u_{2}\left(x_{1}, b_{\mathrm{c}}\right)-u_{2}^{\mathrm{B}} \\
& u_{1}\left(-a_{\mathrm{c}}, x_{2}\right)=u_{1}\left(a_{\mathrm{c}}, x_{2}\right)-u_{1}^{\mathrm{D}} \\
& u_{2}\left(-a_{\mathrm{c}}, x_{2}\right)=u_{2}\left(a_{\mathrm{c}}, x_{2}\right)-u_{2}^{\mathrm{D}}
\end{aligned}
$$

where $u_{i}^{\mathrm{B}}$ and $u_{i}^{\mathrm{D}}$ are the displacements at the two cell corners B and D, respectively, see Fig. 1(b). Force equilibrium is formulated in terms of the surface tractions

$$
\begin{aligned}
& T_{1}\left(x_{1},-b_{\mathrm{c}}\right)=-T_{1}\left(x_{1}, b_{\mathrm{c}}\right) \\
& T_{2}\left(x_{1},-b_{\mathrm{c}}\right)=-T_{2}\left(x_{1}, b_{\mathrm{c}}\right) \\
& T_{1}\left(-a_{\mathrm{c}}, x_{2}\right)=-T_{1}\left(a_{\mathrm{c}}, x_{2}\right) \\
& T_{2}\left(-a_{\mathrm{c}}, x_{2}\right)=-T_{2}\left(a_{\mathrm{c}}, x_{2}\right)
\end{aligned}
$$

The conditions stated in Eqs. (1) and (2) hold for the cell problem in Fig. 1(b). In addition, some conditions for the macroscopic average problem, denoted by ()$^{\mathrm{AV}}$, must also be specified. Denoting the average stresses on the cell by $S_{i j}^{\mathrm{AV}}$ and the average deformation gradients of the cell by $F_{i j}^{\mathrm{AV}}$, these constraints are here chosen to be

$$
\begin{aligned}
& F_{11}^{\mathrm{AV}}=\frac{\Delta_{1}}{2 a_{\mathrm{c}}}\left(u_{1}^{\mathrm{D}}=\Delta_{1}, \text { tension in the } x_{1} \text {-direction }\right) \\
& F_{21}^{\mathrm{AV}}=0 \quad\left(u_{2}^{\mathrm{D}}=0\right) \\
& S_{12}^{\mathrm{AV}}=0
\end{aligned}
$$

and one of

$$
\left\{\begin{array}{l}
S_{22}^{\mathrm{AV}}=0 \quad(\text { Uniaxial plane strain tension }) \\
F_{22}^{\mathrm{AV}}=\kappa F_{11}^{\mathrm{AV}}\left(u_{2}^{\mathrm{B}}=\kappa u_{1}^{\mathrm{D}}, \text { biaxial plane strain tension }\right)
\end{array}\right.
$$

The ratio, $\kappa$, will be specified later. The condition $F_{21}^{\mathrm{AV}}=0$ means that the cell edges initially parallel with the $x_{1}$-axis may curve locally but on the average these edges remain straight and do not rotate. Similarly, $S_{12}^{\mathrm{AV}}=0$ dictates that edges initially parallel to the $x_{2}$-axis may have an average rotation to fulfill equilibrium. This will be illustrated by subsequent results. For the sake of simplicity no average shear load cases will be studied, but it is noted that in-plane shear can for instance be prescribed by a non-zero $u_{1}^{\mathrm{D}}$-value and $u_{1}^{\mathrm{B}}=u_{2}^{\mathrm{B}}=u_{2}^{\mathrm{D}}=0$. In-plane shear has for instance been studied by Vajari et al. [7].

\section{Material Model}

The reinforcement is assumed to be much stiffer than the matrix and consequently modeled as perfectly stiff. Using tensor notation, the displacement field of the matrix is denoted $u_{\alpha}\left(x_{\beta}\right)$, where the Greek indices take the value 1 or 2 , such that the definition of the small strain tensor, $\varepsilon_{\alpha \beta}$, is

$$
\varepsilon_{\alpha \beta}=\frac{1}{2}\left(u_{\alpha, \beta}+u_{\beta}, \alpha\right)
$$

The matrix material is assumed to behave linear elastic with Young's modulus $E_{m}$ and Poisson's ratio $v_{m}$. The stressstrain relation, $\sigma_{\alpha \beta}=\sigma_{\alpha \beta}\left(\varepsilon_{\alpha \beta}\right)$, is given by Hookes law, 
which for plane strain condition is

$$
\begin{gathered}
\sigma_{\alpha \beta}=\frac{E_{m}}{1+v_{m}}\left\{\varepsilon_{\alpha \beta}+\frac{v_{m}}{1-2 v_{m}} \delta_{\alpha \beta} \varepsilon_{\gamma \gamma}\right\} \\
\sigma_{33}=\frac{v_{m} E_{m}}{\left(1+v_{m}\right)\left(1-2 v_{m}\right)} \varepsilon_{\gamma \gamma} ; \quad \sigma_{3 \alpha}=\sigma_{\alpha 3}=0
\end{gathered}
$$

Here, $\delta_{\alpha \beta}$ denotes Kronecker's delta and a repeated index means summation.

Damage of the material is assumed to be caused by two mechanisms: Interfacial debonding and matrix cracking. Both mechanisms are modeled by the multi-linear cohesive law developed in Ref. [16]. Denoting the normal and tangential directions by subscripts $n$ and $t$, respectively, the interfacial tractions, $T_{n}$ and $T_{t}$, depend on the interfacial separations, $u_{n}$ and $u_{t}$, as

$$
\begin{gathered}
T_{n}\left(u_{n}\right)= \begin{cases}\sigma_{\max } \frac{u_{n}}{\delta_{n 1}} & u_{n} \leq \delta_{n 1} \\
\sigma_{\max } \frac{\delta_{n}^{c}-u_{n}}{\delta_{n}^{c}-\delta_{n 1}} & \delta_{n 1}<u_{n} \leq \delta_{n}^{c} \\
0 & u_{n}>\delta_{n}^{c}\end{cases} \\
T_{t}\left(u_{t}\right)= \begin{cases}\tau_{\max } \frac{u_{t}}{\delta_{t 1}} & \left|u_{t}\right| \leq \delta_{t 1} \\
\operatorname{sgn}\left(u_{t}\right) \tau_{\max } \frac{\delta_{t}^{c}-\left|u_{t}\right|}{\delta_{t}^{c}-\delta_{t 1}} & \delta_{t 1}<\left|u_{t}\right| \leq \delta_{t}^{c} \\
0 & \left|u_{t}\right|>\delta_{t}^{c}\end{cases}
\end{gathered}
$$

where $\delta_{n}^{c}$ and $\delta_{t}^{c}$ denote the critical separations in normal and tangential directions, respectively, at which complete debonding has occurred and no further tractions can be transferred over the interface. The maximum stress carried by the interface is denoted $\sigma_{\max }\left(\right.$ for $\left.u_{n}=\delta_{n 1}\right)$ and $\tau_{\max }\left(\right.$ for $u_{t}=\delta_{t 1}$ ) in normal and tangential direction, respectively. The normal and tangential separations are coupled together by the simple fracture criterion [17]

$$
\frac{\mathcal{G}\left(u_{n}^{*}\right)}{\Gamma_{I}}+\frac{\mathcal{G}\left(u_{t}^{*}\right)}{\Gamma_{I I}}=1
$$

Here, $\mathcal{G}\left(u_{n}^{*}\right)$ and $\mathcal{G}\left(u_{t}^{*}\right)$ are the traction-separation work absorbed during fracture in normal and tangential direction, as the separation parameters, $u_{n}$ and $u_{t}$, evolve simultaneously. The work of fracture in pure normal and pure tangential separation are denoted $\Gamma_{I}$ and $\Gamma_{I I}$, respectively.

Contact in relation to interfacial compression, $\delta_{n}<0$, is not a dominant mechanism in this study, but can occur due to Poisson's ratio effects. In the present model friction-less contact is modeled by linear springs as shown in Eq. (7)a.

\section{Numerical Procedure}

Standard FEM cannot conveniently represent arbitrary crack propagation, as the development of stress free crack faces during the loading history are not possible to account for without a significant amount of bookkeeping and remeshing. Alternatively, the A-FEM method can be adopted. The A-FEM has proven to be an effective method to treat strong discontinuities in materials $[18,19]$. Here, only the most important equations will be provided as the full description is given in Ref. [12].

The method is based on the weak form of the equilibrium equations through the principle of virtual work. Accounting for small deformations and the work done by the cohesive interface the principle of virtual work reads [20]

$$
\int_{\Omega} \sigma_{i j} \delta \varepsilon_{i j} \mathrm{~d} \Omega+\int_{\Gamma_{c}}\left(T_{n} \delta u_{n}+T_{t} \delta u_{t}\right) \mathrm{d} S=\int_{S} T_{i} \delta u_{i} \mathrm{~d} S
$$

Here, $\Omega$ is the volume with the surface $S$, and $\Gamma_{c}$ is the surface where the cohesive model is imposed. Virtual quantities are denoted by $\delta$. In principle the A-FEM methodology can be used both in 3D and for other element types (triangular and higher-order), but currently only bi-linear 4-node quadratic plane element are implemented. In Fig. 2 are examples of meshes used. The mesh is highly refined near the reinforcement. In the case of no discontinuity the A-FEM is identical to standard FEM. However, when a crack is present a strong discontinuity exists. Matrix cracking initiates, when the average element stress reaches the critical stress of the matrix, $\sigma_{\text {max }}$ in Eq. (7). Then, a cohesive zone is activated, as illustrated in Fig. 3, in the direction perpendicularly to the maximum principal stress, such that the crack grows according to the maximum principal stress criterion. An additional set of nodes (shown by the numbers with primes) are used to form two mathematical elements (ME1 and ME2), which constitutes the physical standard element (SE), i.e. $\Omega^{e}=\Omega_{1}^{e} \cup \Omega_{2}^{e}$. Only two elements are formed as the algorithm only allows for crack tips arrested at element edges. Both MEs have identical geometry as the SE, but with different material allocation, as shown in 3(b)-(c). The essential part in A-FEM is the mapping of the standard shape functions, $\mathbf{N}_{\alpha}$, in each $\mathrm{SE}$ to the corresponding $\mathrm{ME}$

$$
\mathbf{N}_{\alpha}^{\prime}=\mathbf{N}_{\alpha} \cdot \mathbf{T}_{\alpha}
$$

where $\alpha=1,2$ indicates the two elements formed when an element is traversed by a discontinuity. The mapping matrix, $\mathbf{T}_{\alpha}$, for this 4-node quadratic plane element is a $8 \times 8$ matrix and an example is provided by [12]. Within each ME the displacements, $\mathbf{u}_{\alpha}^{\prime}$, can then be found in the usual manner using the transformed shape functions and the nodal displacements, $\mathbf{d}_{\alpha}^{\prime}$

$$
\mathbf{u}_{\alpha}^{\prime}=\mathbf{N}_{\alpha}^{\prime} \cdot \mathbf{d}_{\alpha}^{\prime}
$$

This is inserted into the principle of virtual work to form the new element stiffness matrix, $\mathbf{k}_{\alpha}^{\prime}$, the new consistent element 


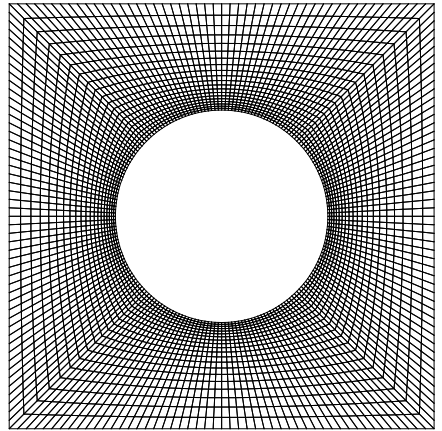

a)

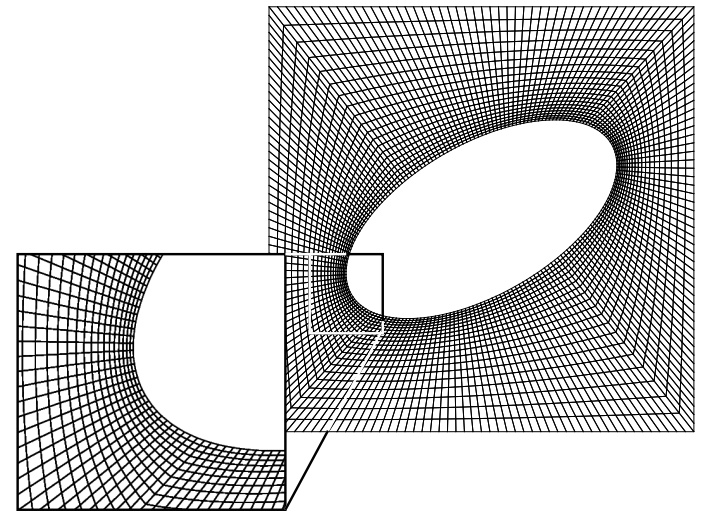

b)

Fig. 2. Examples of finite element meshes used. (a) $a_{\mathrm{i}} / b_{\mathrm{i}}=a_{\mathrm{c}} / b_{\mathrm{c}}=1$. (b) $a_{\mathrm{i}} / b_{\mathrm{i}}=2$ and $a_{\mathrm{c}} / b_{\mathrm{c}}=1$ for $\beta_{\mathrm{i}}=30^{\circ}$. A magnification near the reinforcement is inserted.

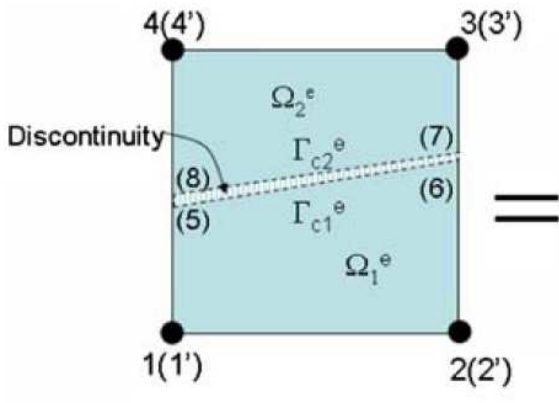

a)

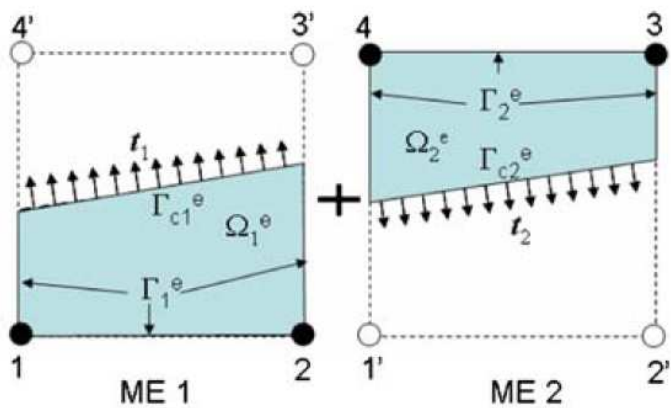

b)

c)

Fig. 3. Representation of a matrix crack using A-FEM [12]. a) Cracked element in domain $\Omega^{e}=\Omega_{1}^{e}+\Omega_{2}^{e}$. b) Mathematical element of domain $\Omega_{1}^{e}$ (ME1). c) Mathematical element of domain $\Omega_{2}^{e}$ (ME2).

nodal load vector resulting from the surface tractions, $\overline{\mathbf{f}}_{\alpha}^{\prime}$, and lastly the nodal load vector resulting from the cohesive tractions, $\mathbf{f}_{\alpha}^{\prime}$. The system of linear equations on the element level is then

$$
\mathbf{k}_{\alpha}^{\prime} \mathbf{d}_{\alpha}^{\prime}=\overline{\mathbf{f}}_{\alpha}^{\prime}+\mathbf{f}_{\alpha}^{\prime}
$$

This is then assembled in the usual way to generate the global system of equations

$$
\mathbf{K D}=\overline{\mathbf{F}}+\mathbf{F}
$$

that can be solved for the nodal displacements, D, after appropriate boundary conditions are introduced. From the displacement field can the strains and stresses be obtained in the usual way for FEM.

Finally, the cohesive law in Eqs. (7) and (8) results in a stiffness contribution as can be seen from Eq.(10), such that interfacial separation as well as contact are implemented in a manner similar to a standard penalty method. The frictionless contact is modeled by the mode I cohesive law, which provides a large stiffness to minimize interpenetration, but the resulted compressive normal stress does not introduce any additional shear stress in the tangential direction.

\section{Numerical Results}

In this study focus will be on damage with a fixed reinforcement volume fraction of $20 \%$ and $\sqrt{a_{\mathrm{i}} b_{\mathrm{i}}}=5 \cdot 10^{-3}$ $\mathrm{mm}$. The elastic material parameters used for the matrix material are $E_{m}=4 \mathrm{GPa}$ and $v_{m}=0.33$. The ratio between the strength of the matrix and the interface defines the failure mode. If the interface strength is small compared to the matrix, failure is dominated by interface debonding. On the other hand, if the interface strength is large compared to the matrix, failure is dominated by matrix cracking. Assuming isotropy, matrix fracture is modeled using the A-FEM method with the same cohesive parameters in normal as well as tangential separation, i.e. $\sigma_{\max }=\tau_{\max }=100$ $\mathrm{MPa}, \delta_{n 1}=\delta_{t 1}=0.01 \delta^{c}$ with $\delta^{c}=\delta_{n}^{c}=\delta_{t}^{c}=0.02 \sqrt{a_{\mathrm{i}} b_{\mathrm{i}}}$, see Eqs. (7)-(8). Interfacial debonding is modeled using the same cohesive parameters except that $\sigma_{\max }=\tau_{\max }=60 \mathrm{MPa}$, which makes the interface the weakest part. Hence, failure 


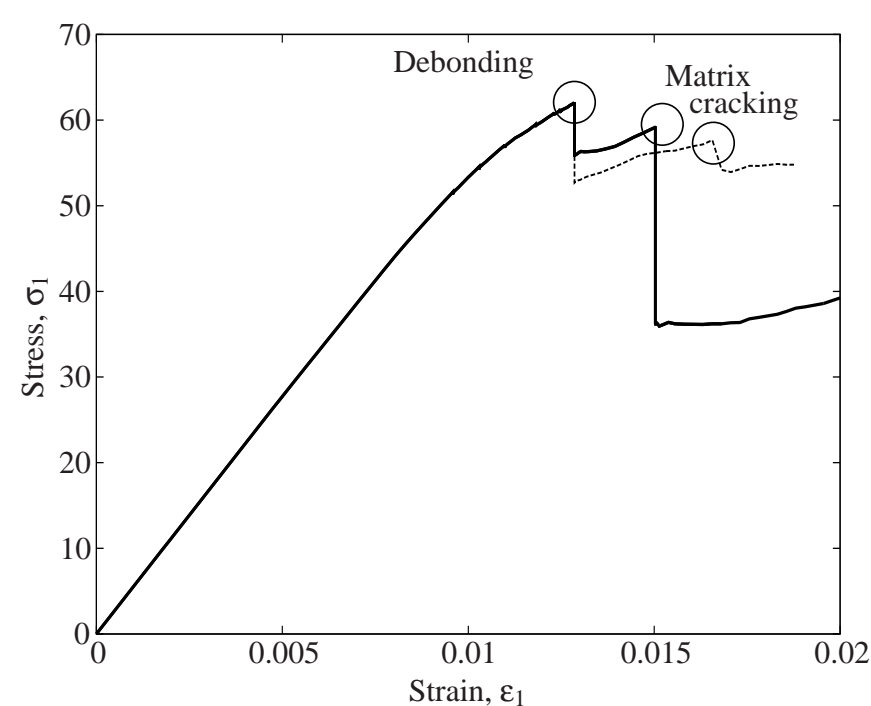

Fig. 4. Stress-strain curves for $a_{\mathrm{i}} / b_{\mathrm{i}}=a_{\mathrm{c}} / b_{\mathrm{c}}=1$ for two different sets of strength parameters. The ratio of the matrix to interface strength is 40 times larger for the dashed line compared to that of the solid line.

is expected to initiate from interfacial debonding and then subsequently propagating into the matrix. The strength of the interface is assumed to be homogeneous along the interface, which means that due to the symmetries of the problem, see Fig. 1, the exact location of the debonding initiation is not known in advanced but is determined by the numerics. Alternatively, an inhomogeneous interfacial strength can be prescribed, such that the exact location of the debonding initiation is predetermined [21].

In the first study a uniform distribution of circular reinforcement is considered, i.e. $a_{\mathrm{i}} / b_{\mathrm{i}}=a_{\mathrm{c}} / b_{\mathrm{c}}=1$. Uniaxial plane strain tension is considered, see Eq. (4)a, and the overall stress-strain response is extracted as

$$
\sigma_{1}=\frac{1}{2 b_{\mathrm{c}}} \int_{-b_{\mathrm{c}}}^{b_{\mathrm{c}}}\left[T_{1}\right]_{x_{1}=a_{\mathrm{c}}} \mathrm{d} x_{2} \quad ; \quad \varepsilon_{1}=\frac{\Delta_{1}}{2 a_{\mathrm{c}}}
$$

Note, that since small deformations are considered here is $\sigma_{1}=S_{11}^{\mathrm{AV}}$ and $\varepsilon_{1}=F_{11}^{\mathrm{AV}}$, Eqs. (3) and (4). Two different cases of different strength are studied, see Fig. 4, both resulting in initial interfacial debonding followed by matrix cracking. Hence, in addition to the above mentioned strength parameters (solid line in Fig. 4) a second case with a matrix to interface strength ratio 40 times larger is also consider (dashed line in Fig. 4). In this case the interfacial strength is unchanged, while the matrix strength is assumed 40 times stronger by keeping $\sigma_{\max }=\tau_{\max }=100 \mathrm{MPa}$ and increasing $\delta_{n 1}=\delta_{t 1}=0.01 \delta^{c}$ with $\delta^{c}=\delta_{n}^{c}=\delta_{t}^{c}=0.8 \sqrt{a_{\mathrm{i}} b_{\mathrm{i}}}$.

A sudden stress drop in these curves represents failure. It is seen from Fig. 4 that the cases result in identical responses up to interfacial debonding at $\varepsilon_{1} \simeq 0.013$ at a stress level corresponding to $\sigma_{\max }=60 \mathrm{MPa}$. Some non-linearity is observed, which is related to the cohesive law. The case with the weaker matrix (solid line) shows the smaller stress
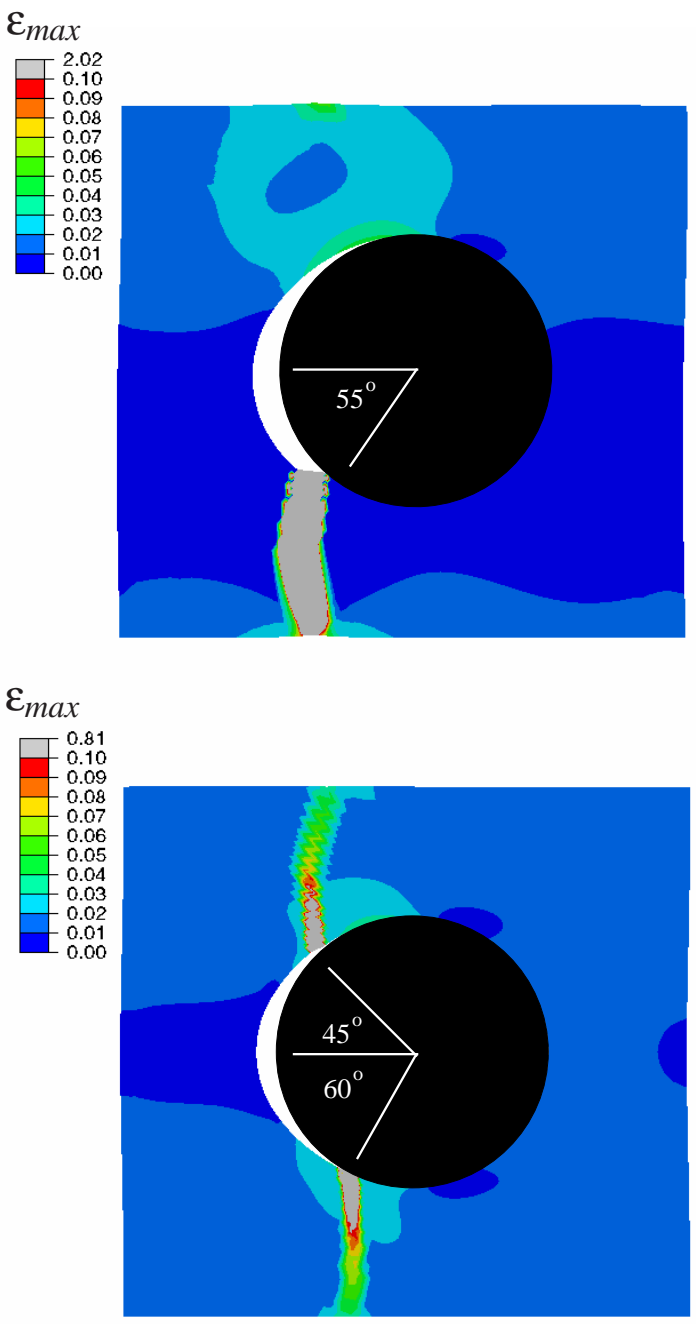

a)

b)

Fig. 5. Contours of maximum principal strain, $\varepsilon_{\text {max }}$, at $\varepsilon_{1}=0.018$ in Fig. 4. Matrix cracks are shown by gray color and the deformations are 3 times magnified. a) Low matrix to interface strength ratio (solid line in Fig. 4). b) 40 times larger matrix to interface strength ratio (dashed line in Fig. 4).

drop as a result of interfacial debonding. By further loading a second stress drop occur, which is caused by matrix cracking. The relatively stronger matrix (dashed line) is failing at $\varepsilon_{1} \simeq 0.017$, while the weaker one fails at $\varepsilon_{1} \simeq 0.015$. The latter shows a large and sudden (unstable) stress drop, whereas the other is more moderate and less steep. This less steep stress drop results from the fact, that the 40 times larger strength ratio is obtained by having a larger critical separation, $\delta^{c}$, of the matrix, which stabilize the degradation. Fig. 5 shows the corresponding contours of the maximum principal strain, $\varepsilon_{\max }$, at the overall strain level $\varepsilon_{1}=0.018$ in Fig. 4. The deformations are magnified 3 times. The debond at the interface is seen as the white void nucleated along the left side of the rigid circular reinforcement, which is shown by black color. The matrix crack is shown by gray color. As discussed above, the fact that the location of the nucleated 
void is to the left is a result of the symmetry of the problem as well as the numerics. Fig. 5(a) corresponds to the solid curve in Fig. 4 and Fig. 5(b) corresponds to the dashed curve in Fig. 4. At this level of overall straining the cracking of the matrix has occurred in quite different ways. Fig. 5(a) shows a single matrix crack evolving from the tip of the interface crack in a $55^{\circ}$ direction relatively to the loading, while Fig. 5 (b) indicates that two matrix cracks nucleate. One of these are at the lower part of the debond at practically the same location $\left(60^{\circ}\right)$ as in Fig. 5(a). However, a second matrix crack initiates almost simultaneously at the tip of the upper part as well, Fig. 5(b). The angle of this crack is only $45^{\circ}$. Paris et al. and Ashouri found experimentally, that the angle at which the crack kinks in to the matrix is approximately $60^{\circ}-70^{\circ}$ which in comparison with the single matrix crack observations of Fig. 5(a) is in rather good agreement [22,23]. Hence, for the subsequent analyses, these strength parameters are adopted, see above.

As discussed in the introduction, an inherent feature of the single inclusion cell in Fig. 5 is, that the strain field near all neighboring inclusions are identical to the one analyzed, resulting in the exact same angle of matrix cracking. In real materials are both the distribution of the second phase as well as the interfacial bonding often a complex (or even a random) function of spacial coordinates. In Fig. 6 is the effect of rigidly bonded inclusions surrounding a weaker bonded inclusion investigated. A mesh similar to Fig. 2(a) is adopted, but extended to a $3 \times 3$ configuration, such that eight cells containing rigidly bonded inclusions surround the central debonding one. If all inclusions were modeled to be able to debond, then the single cell studies is expected to be reproduced from the $3 \times 3$ configuration. Uniaxial tension and periodic boundary conditions are still specified and all material parameters are identical to those in Fig. 5(a). It is seen, that the strain field is disturbed to the left and to the right of the debond, but fairly unaffected above and below. The angle between the loading direction and the matrix crack is still found to be approximately $55^{\circ}$ as in Fig. 5(a). However, the overall strain is reduced to $\varepsilon_{1} \simeq 0.010$ from $\varepsilon_{1} \simeq 0.018$ in Fig. 5(a). For an even stronger matrix material the same overall strain of $\varepsilon_{1} \simeq 0.010$ results in two matrix cracks evolve symmetrically at slightly smaller angles similar to Fig. 5(b) (results not shown here). Thus, the interference with other fibers for this fiber volume fraction is rather low for the microscopic result of the kinking angle and consequently is a single cell sufficient. It is noticed, that the matrix crack in Fig. 6 grows in the upward direction, whereas the crack propagates downwards in the corresponding single cell, Fig. 5(a). As discussed above this is caused by the numerics and due to the symmetries of the problem. Initially, are both the load, the material and strength distribution symmetric around the dashed line shown in Fig. 6, meaning that both tips of the interfacial debond are potential nucleation sites for the matrix cracks. In the A-FEM analysis, each load increment permits only one crack initiation, i.e. the element with the largest average stress that exceeds the cohesive strength. Thus, in a symmetric model with symmetric loading, even if more than one elements are expected to initiate

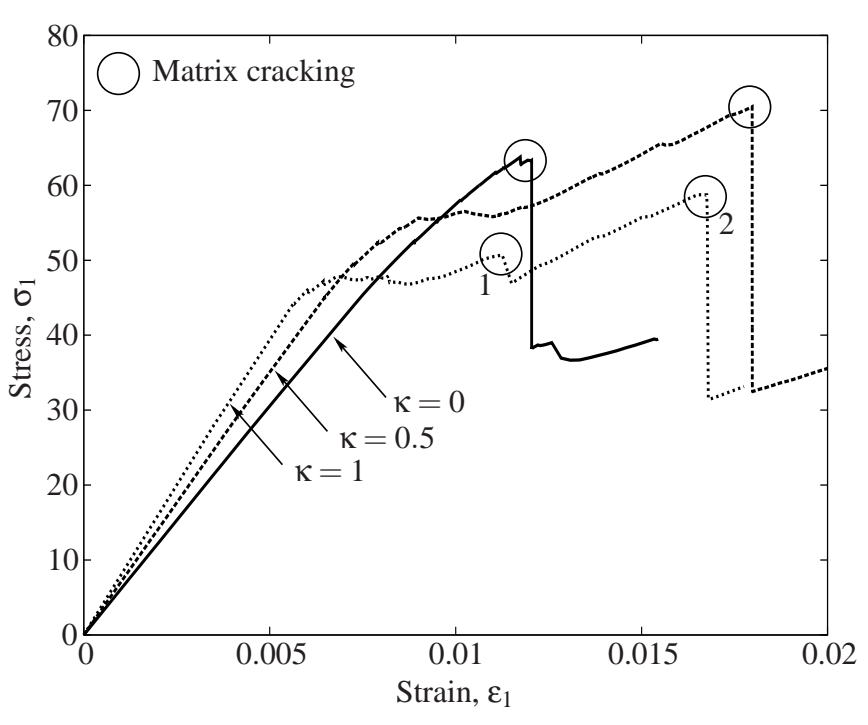

Fig. 7. Stress-strain curves for $a_{\mathrm{i}} / b_{\mathrm{i}}=a_{\mathrm{c}} / b_{\mathrm{c}}=1$ for three different values of the loading parameter, $\kappa$, see Eq. (4)b.

simultaneously according to theoretical predictions, the AFEM will choose only one of them to initiate, which could be determined by numerical truncation errors, the order of element numbering, or local mesh variance. After the initiation, the model symmetry is broken. This is responsible for the asymmetric crack development observed. We kept this feature in the A-FEM because in reality perfectly symmetric crack development are rarely observed due to local material inhomogeneity at micro-meso scales.

For biaxial plane strain tension, see Eq. (4)b, Fig. 7 gives the overall stress-strain response in the $x_{1}$-direction for the same parameters as in Figs. 4 (solid line) and 5(a). The most important parameters characterizing the fracture process are the fracture energy and the peak stress for the traction-separation law [24]. The shape of the tractionseparation law defined through $\delta_{n 1}$ and $\delta_{t 1}$ is of less importance and should be taken as small as possible to reduce artificial initial stiffness. However, for the cases with $\kappa>0$ $\delta_{n 1}=\delta_{t 1}=0.05 \delta^{c}$ have been used to stabilize the numerical solution. Three different loading cases are considered, namely plane-plane strain tension $(\kappa=0$ with no average contraction in the $x_{2}$-direction), biaxial plane strain tension $(\kappa=0.5)$ and equi-biaxial plane strain tension $(\kappa=1)$. It is noted, that all three cases may introduce an overall stress component different from zero in the $x_{2}$-direction, i.e. $\sigma_{2} \neq$ 0 . A larger stiffness is predicted for increasing $\kappa$-values. The point at which interfacial debonding starts is here seen as the point where the curves start to deviate from linearity but no sudden stress drop is detected during debonding. On the other hand, matrix cracking does show significant stress drops. These are highlighted by circles on the curves. The case of $\kappa=1$ fails at the lowest stress-level and hereafter the level of stress at failure increases for decreasing $\kappa$-values. This is expected, as high $\kappa$-values introduce a non-zero $\sigma_{2}$ which assists in failing the matrix. An important observation is, that for $\kappa=1$ the material fails by forming two matrix 


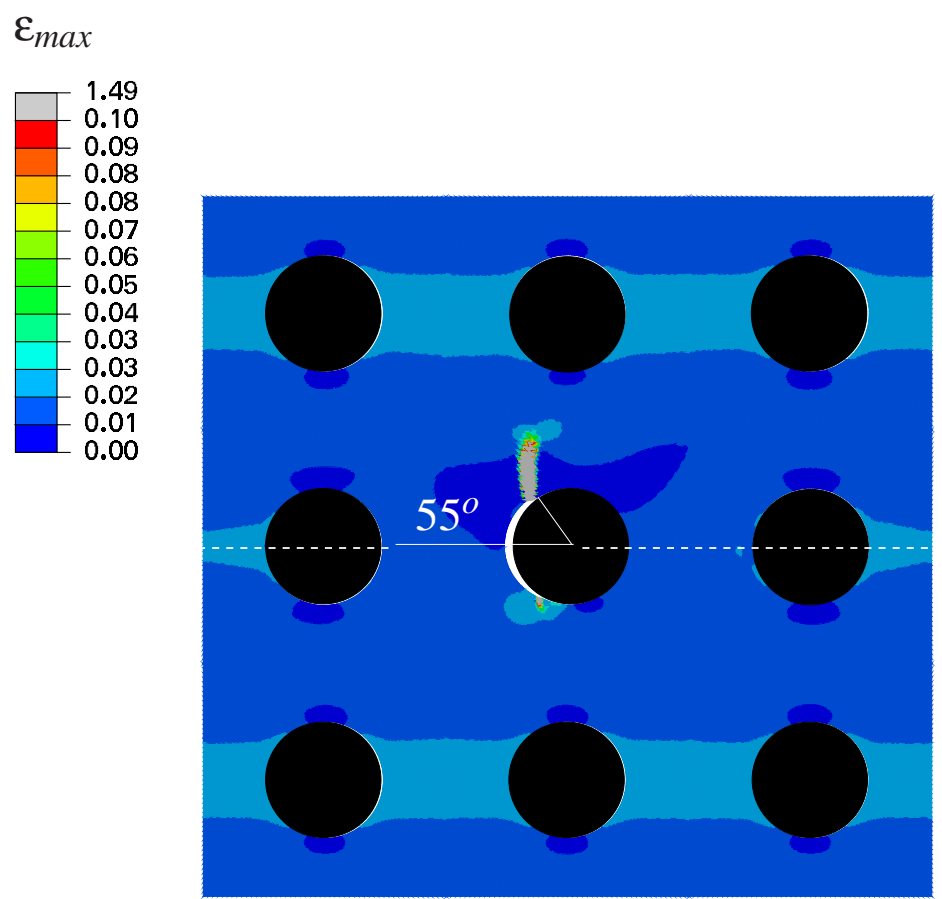

Fig. 6. Contours of maximum principal strain, $\varepsilon_{\max }$, at $\varepsilon_{1}=0.010$. Matrix crack is shown by gray color and the deformations are 3 times magnified. Only the central reinforcement is allowed to debond.

cracks, which does not happen at the lower triaxialities, i.e. at $\kappa=0$ and $\kappa=0.5$. The first matrix crack, which is marked by "1", happens before the matrix crack for $\kappa=0$ initiates, but the second matrix crack, marked by "2", starts after. The case of $\kappa=0.5$ fails by matrix cracking at the largest strain level, $\varepsilon_{1} \simeq 0.018$.

The corresponding contours of the maximum principal strain in the deformed cell for $\kappa=0$ are similar to what is shown for the uniaxial plane strain tensile case, Fig. 5(a), and will not be shown here. This is expected, as the stress-strain curves are quite similar (as seen by comparison of solid lines of Figs. 4 and 7). Fig. 8 shows the contours of the maximum principal strain, $\varepsilon_{\max }$, for the two other cases, i.e. $\kappa=0.5$ and $\kappa=1$, which both have a significantly different stress-strain response compared to that of $\kappa=0$, see Fig. 7. Fig. 8(a) is for $\kappa=0.5$ and Fig. 8(b) is for $\kappa=1$ and the deformations are 3 times magnified. Despite the fact that the stress-strain response for $\kappa=0.5$ is very different to that of $\kappa=0$ as well as that for uniaxial plane strain tension, the crack paths in the matrix are very similar. A single crack evolves from the tip of the debonding crack and propagate to the edge of cell, Fig. 8(a). The direction of the crack is slightly affected by the loading condition, as it is not perpendicular to the principal loading direction of the cell, the $x_{1}$-direction, as was seen in Fig. 5(a). Furthermore the dashed lines in Fig. 8(a) also illustrate the conditions stated in Eq. (3)b-c. The cell edges initially parallel with the $x_{1}$-axis curve locally but on the average these edges remain straight and cell edges initially parallel with the $x_{2}$-axis rotate on the average to fulfill $S_{12}^{\mathrm{AV}}=0$. For the largest triaxiality, $\kappa=1$, Fig. $8(\mathrm{~b})$ shows that two cracks have evolved from each tip of the debond. Their directions are nearly the same and form an angle of approximately $60^{\circ}$ relatively to the $x_{1}$-direction, which is most clearly seen for the lower matrix crack.

The volume fraction of reinforcement is kept constant at 20\% and Figs. 9-12 provide the effects of geometrical anisotropy under uniaxial plane strain tension, see Eq. (4)a. Geometrical anisotropy exists if the distribution of the reinforcement is different in the $x_{1}$-direction to that of the $x_{2}$ direction $\left(a_{\mathrm{c}} / b_{\mathrm{c}} \neq 1\right)$ or the cross section of the inclusion is elliptical $\left(a_{\mathrm{i}} / b_{\mathrm{i}} \neq 1\right)$ and/or inclined relatively to the cell edges. Figs. 9-10 study the case of a non-uniform distribution of circular reinforcement, i.e. $a_{\mathrm{i}} / b_{\mathrm{i}}=1$ for $a_{\mathrm{c}} / b_{\mathrm{c}} \neq 1$, while Figs. 11-12 treat $a_{\mathrm{i}} / b_{\mathrm{i}}=2$ with $a_{\mathrm{c}} / b_{\mathrm{c}}=1$ for different orientations, $\beta_{\mathrm{i}}$, see Fig. 1(b).

In Fig. 9 is the geometrically isotropic case, $a_{\mathrm{i}} / b_{\mathrm{i}}=$ $a_{\mathrm{c}} / b_{\mathrm{c}}=1$ (solid line), repeated from Fig. 4 (solid line). The three cases considered are illustrated by small insertions. Up to an overall strain of $\varepsilon_{1} \simeq 0.01$ are the responses very similar with a tendency of being slightly more non-linear for the case $a_{\mathrm{c}} / b_{\mathrm{c}}=\frac{1}{2}$. Changing the spacing of the reinforcement, i.e. $a_{\mathrm{c}} / b_{\mathrm{c}}$, clearly affects both the failure strain and the load carrying capacity. For an increasing $a_{\mathrm{c}} / b_{\mathrm{c}}$ ratio is the load carrying capacity also improved. However, the ductility (the failure strain) is largest for $a_{\mathrm{c}} / b_{\mathrm{c}}=1$ and is clearly defined by matrix cracking, see also Fig. 4 (solid line). The largest drop in stress is seen for $a_{\mathrm{c}} / b_{\mathrm{c}}=2$ at $\varepsilon_{1} \simeq 0.014$. The configuration $a_{\mathrm{c}} / b_{\mathrm{c}}=\frac{1}{2}$ shows the worst response both in terms of ductility and load carrying capacity. Eventually is the load carrying capacity practically the same for the three 


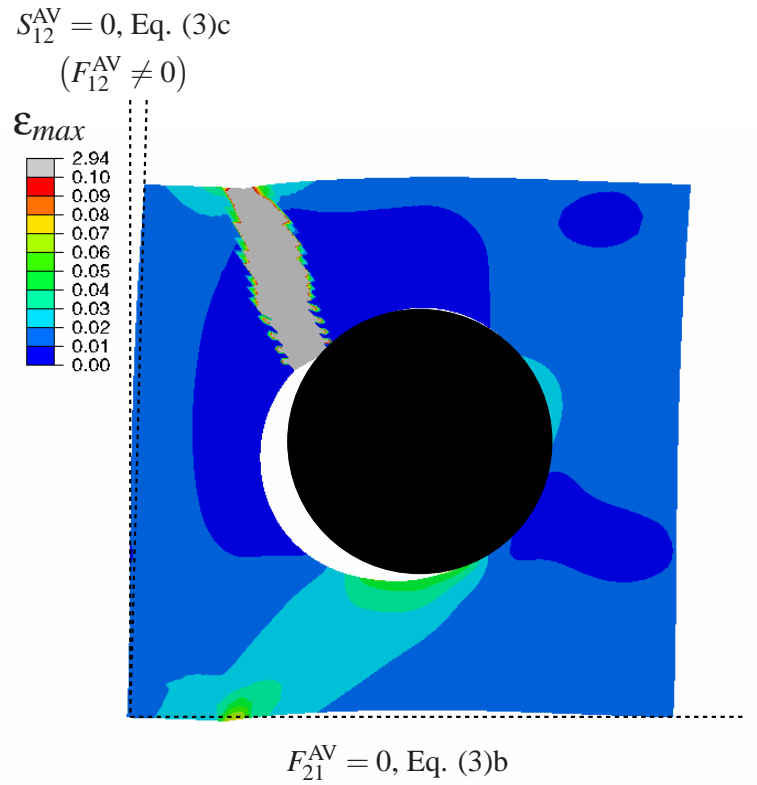

a)

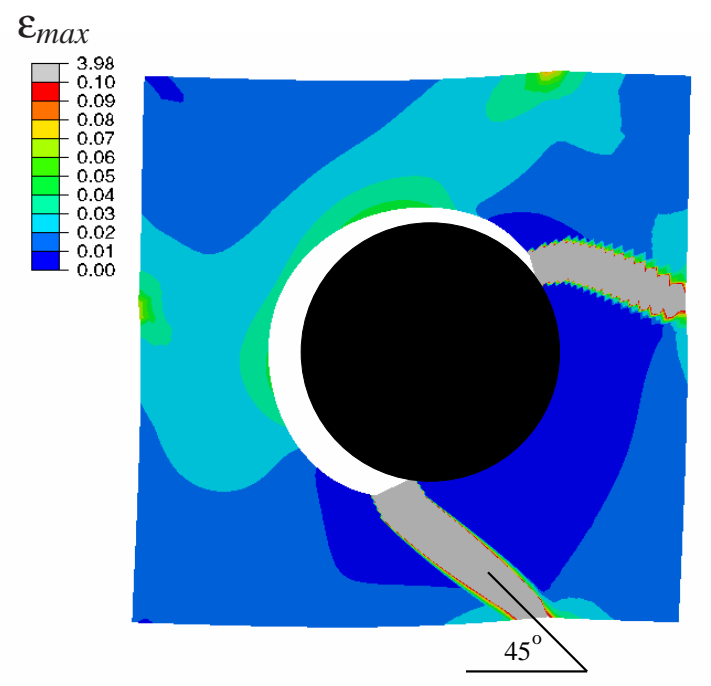

b)

Fig. 8. Contours of maximum principal strain, $\varepsilon_{\max }$, at $\varepsilon_{1}=0.018$ in Fig. 7. Matrix cracks are shown by gray color and the deformations are 3 times magnified. a) Biaxial loading, $\kappa=0.5$. The conditions in Eq. (3)b-c are also illustrated. b) Equi-biaxial loading, $\kappa=1$.

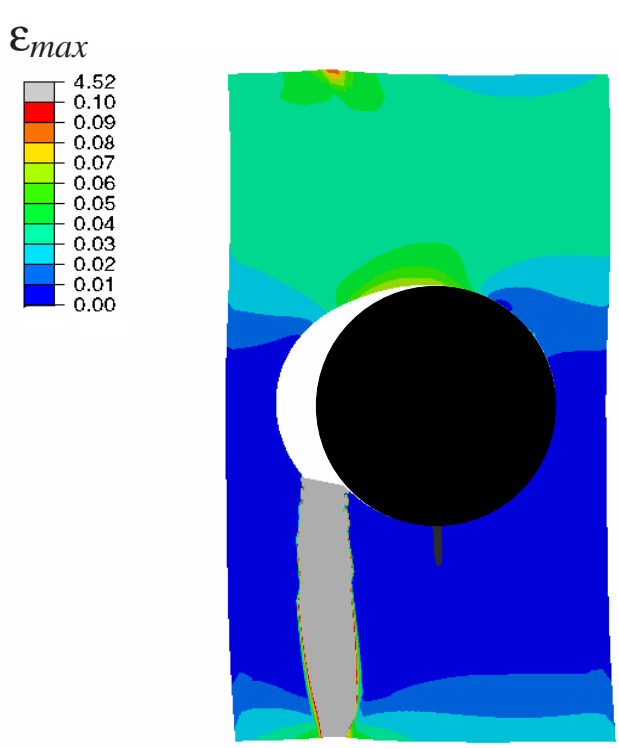

a)

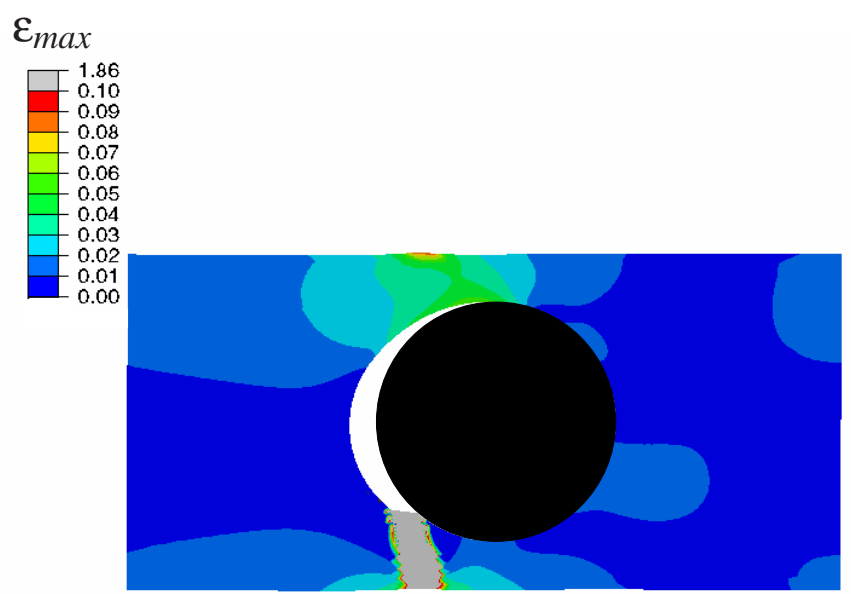

b)

Fig. 10. Contours of maximum principal strain, $\varepsilon_{\max }$, at $\varepsilon_{1}=0.018$ in Fig. 9. Matrix cracks are shown by gray color and the deformations are 3 times magnified. a) $a_{\mathrm{c}} / b_{\mathrm{c}}=\frac{1}{2}$. b) $a_{\mathrm{c}} / b_{\mathrm{c}}=2$.

cases studied, i.e. for $\varepsilon_{1} \gtrsim 0.015$.

In Fig. 10 are the deformed cells of the two geometrically anisotropic cases of Fig. 9, $a_{\mathrm{c}} / b_{\mathrm{c}}=\frac{1}{2}$ and $a_{\mathrm{c}} / b_{\mathrm{c}}=2$ at $\varepsilon_{1}=0.018$, shown with contours of the maximum principal strain, $\varepsilon_{\max }$. The deformations are 3 times magnified. The matrix cracks are initiated at the tip of the void and they grow to the lower edges of the cell perpendicular to the loading direction. At this stage of loading are the deformations intensified in the upper part of the cells, where there is no matrix crack. It can also be seen from the contours, that the crack is about to propagated from the top cell edge down to the reinforcement, which will eventually define the ultimate strength of the material. This holds true for both $a_{\mathrm{c}} / b_{\mathrm{c}}=\frac{1}{2}$ and $a_{\mathrm{c}} / b_{\mathrm{c}}=2$.

Lastly, non-circular cross-section of the reinforcement, $a_{\mathrm{i}} / a_{\mathrm{i}}=2$, are investigated. Non-conventional cross sections such as triangular, square with holes, cruciform and pentalobal have been studied in Refs. [25, 26]. A fiber with a non-circular cross section has a higher surface area over volume ratio, compared with that having a circular cross sec- 


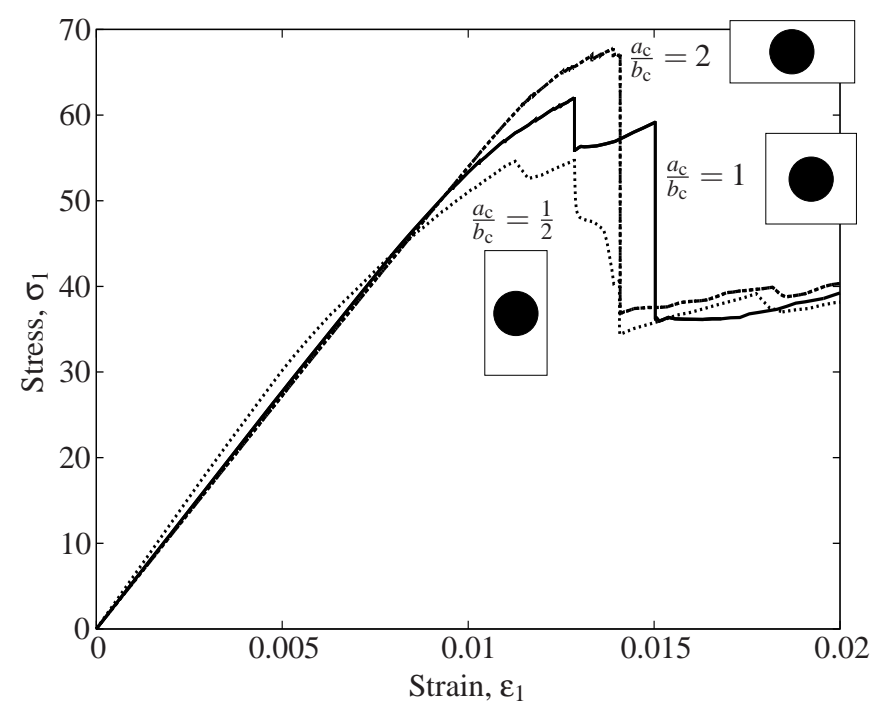

Fig. 9. Stress-strain curves for $a_{\mathrm{i}} / b_{\mathrm{i}}=1$ for three different values of $a_{\mathrm{c}} / b_{\mathrm{c}}$ under uniaxial plane strain tension.

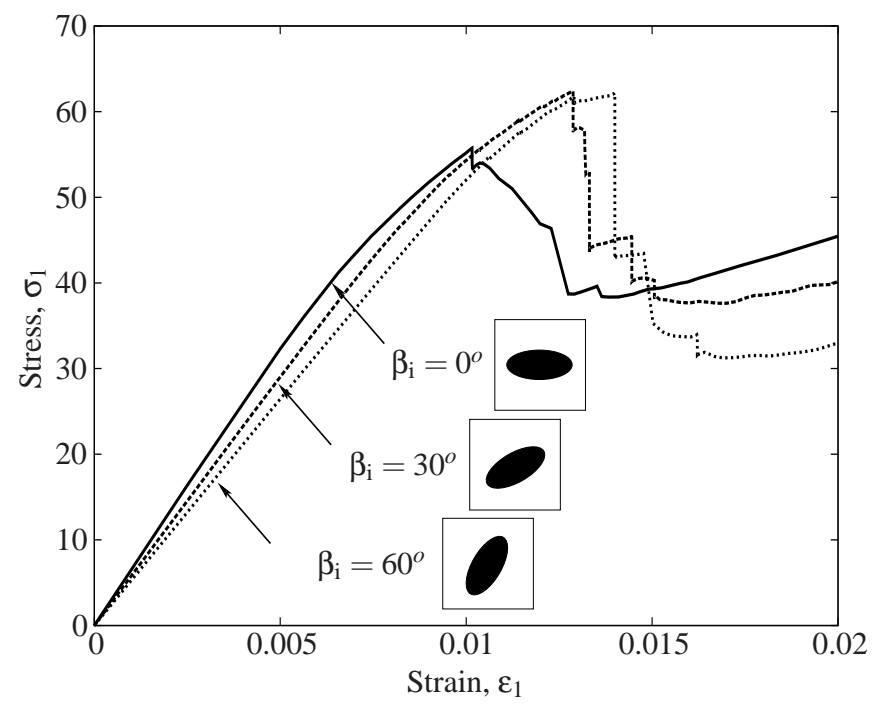

Fig. 11. Stress-strain curves for $a_{\mathrm{i}} / b_{\mathrm{i}}=2$ and $a_{\mathrm{c}} / b_{\mathrm{c}}=1$ for three different values of the orientation, $\beta_{\mathrm{i}}$.

tion, as discussed by [27]. Here, $a_{\mathrm{i}} / b_{\mathrm{i}}=2$ yields a $6 \%$ increase over the circular case. Non-circular SiC fibres have a higher tensile strength than that of the circular SiC fibres and furthermore exhibit an excellent microwave-absorbing property [28]. The stress-strain curves for three different angles, i.e. $\beta_{\mathrm{i}}=0^{\circ}, \beta_{\mathrm{i}}=30^{\circ}$ and $\beta_{\mathrm{i}}=60^{\circ}$ are given in Fig. 11 . The three cases are also illustrated by small insertions. A slightly stiffer response is predicted initially for decreasing $\beta_{\mathrm{i}}$-values, such that $\beta_{\mathrm{i}}=0^{\circ}$ yields the highest stiffness and $\beta_{\mathrm{i}}=60^{\circ}$ results in the lowest stiffness. This is believed to be related to the finite stiffness introduced at the interface due to the cohesive law. For $\beta_{\mathrm{i}}=60^{\circ}$ is a large fraction of the interface in the direction of the load, and this causes the effective overall stiffness to be smallest. Focusing on the parts of the curves that represent damage, $0.01 \lesssim \varepsilon_{1} \lesssim 0.015$, it can be seen, that a larger $\beta_{\mathrm{i}}$-value has a positive impact on the curves. Both the strain at which failure is occurring as well at the maximum stress increases for increasing $\beta_{\mathrm{i}}$-values. Furthermore, the sudden stress drop observed for $\beta_{i}=60^{\circ}$ becomes less steep for $\beta_{\mathrm{i}}=30^{\circ}$ and even more smooth for $\beta_{\mathrm{i}}=0^{\circ}$. This reflects a mechanically more stable degradation. The largest drop in stress is for $\beta_{\mathrm{i}}=60^{\circ}$ (approximately $50 \%$ from $\sigma_{1} \simeq 60$ $\mathrm{MPa}$ to $\sigma_{1} \simeq 30 \mathrm{MPa}$ ) and well hereafter, $\varepsilon_{1} \gtrsim 0.015$, is the load carrying capacity largest for $\beta_{\mathrm{i}}=0^{\circ}$ and smallest for $\beta_{\mathrm{i}}=60^{\circ}$.

Finally are the contour plots of the maximum principal strain, $\varepsilon_{\max }$, for all three cases of Fig. 11 provided in Fig. 12. The deformations are 3 times magnified. The contours are very similar to the previous ones, where the matrix crack forms at the tip of the interface crack. Furthermore, for all three values of the orientation angle, $\beta_{\mathrm{i}}$, it can be observed, that the matrix crack starts at the narrow end of the elliptical reinforcement and propagates to the edge in a direction perpendicular to the applied load.

\section{Conclusion}

Failure of dual-phase materials is a field that has attracted a lot of attention over the years due to the existence of a relatively large number of different failure modes. Experiments have shown that for uniaxial transverse tension failure often occurs as interfacial debonding followed by matrix cracking. These two failure mechanisms are numerically investigated in the present study.

A 2D unit cell approach with periodical boundary condition is used to represent perfectly stiff reinforcement embedded in a purely elastic material. A cohesive zone approach is adopted along the interface of the reinforcement, while the A-FEM method is used to capture arbitrarily matrix cracking. The strength properties are chosen such that the interface of the reinforcement fails before the matrix. Hence, a crack starts at the interface and eventually kinks into the matrix. The ratio between the matrix strength and the interfacial strength defines when this kinking initiates. However, even for a 40 times larger ratio the angle relatively to the loading direction of the kink is only changed by approximately $5^{\circ}$. [22] found experimentally, that the angle at which the crack kinks into the matrix is approximately $60^{\circ}-70^{\circ}$, which is in rather good agreement with the simulations, Fig. 5. Even if multiple reinforcements are considered the angle is practically unaffected.

Bi-axial loading has also been studied. In the load range investigated only the case of fully equi-biaxial loading makes the interface debond kink into the matrix by forming two matrix cracks. These are positioned at each tip of the debond. However, by further loading cases with lower triaxialities may also eventually fail by two matrix cracks. The geometrically anisotropic cases of elliptical reinforcement cross sections all show, that the crack kinks out at the narrow end of the ellipse. This is seen for all reinforcement orientations studied, see Fig. 12. 

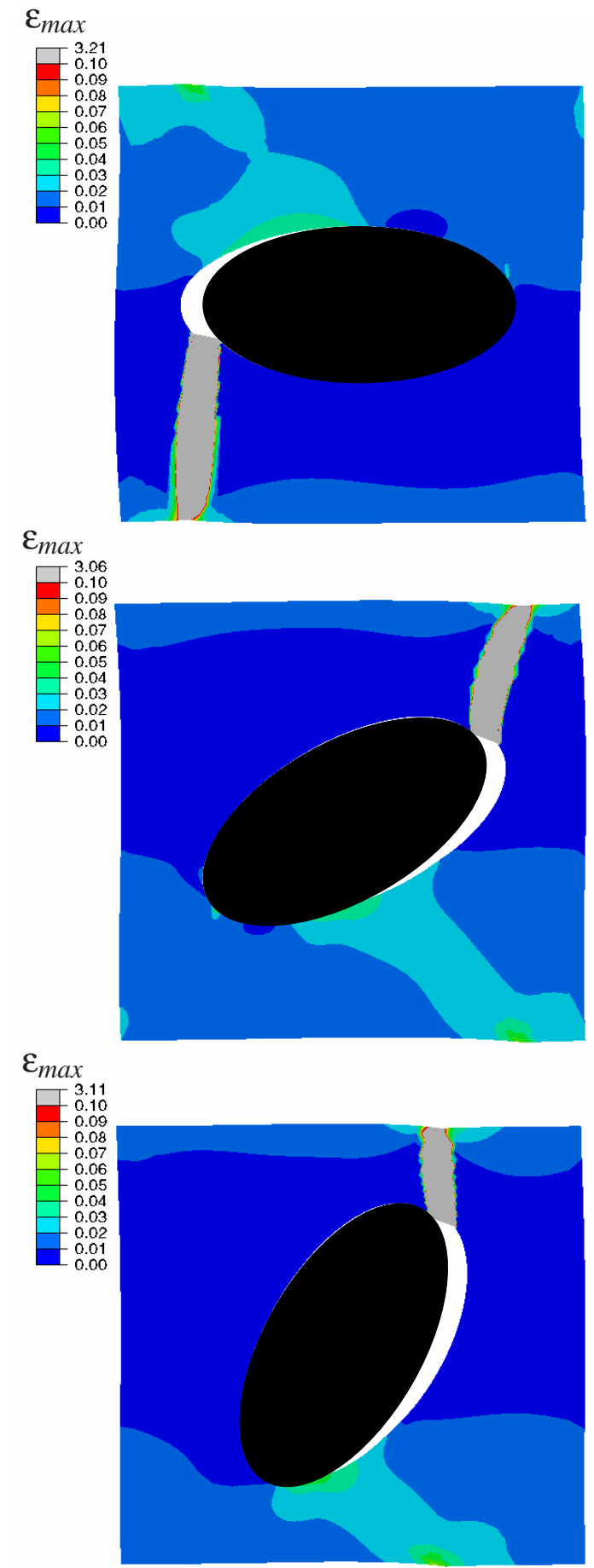

b)

Fig. 12. Contours of maximum principal strain, $\varepsilon_{\max }$, at $\varepsilon_{1}=0.018$ in Fig. 11. Matrix cracks are shown by gray color and the deformations are 3 times magnified. a) $\beta=0^{\circ}$. b) $\beta_{\mathrm{i}}=30^{\circ}$. c) $\beta_{\mathrm{i}}=60^{\circ}$.

\section{Discussion}

For the sake of simplicity, isotropy has been assumed for the constituents throughout the paper. Whereas the elastic properties are most often isotropic, the fracture energies may for some materials, e.g. epoxy, be different in normal and tangent separation. The effects of having a larger normal strength compared to the tangential strength have been studied. The main difference was seen, when two cracks are formed, for instance when $\kappa>0$, Fig. 8. The first crack to initiate is practically not affected, while the second crack may form at another location away from the tip of the debond. For $\kappa=0$, Fig. 5, it is observed that a second crack forms at a lower overall deformation, but the first crack is basically unaffected.

The present work ignores the effect of matrix plasticity. Including plasticity is a non-trivial task and may require the use of more advanced fracture mechanics models. The Embedded Process Zone (EPZ) [24, 29, 30] uses a cohesive model as an interface boundary condition between adjacent solid elements. This technique suffers from being meshdependent, but has the advantage of not necessarily knowing the crack path in advance. The SSV-model introduces an elastic zone near the crack tip [31]. Within this zone standard Linear Elastic Fracture Mechanics (LEFM), where the energy release rate should attain the work of fracture, is used. However, the technique requires the location of the crack tip to be known a prior. If plasticity effects were accounted for a better agreement between experiments and the numerically simulated matrix crack angle might be achieved. Plasticity reduces the stress level for the same level of deformation, or equivalently increases the deformation at the same level of stress. Hence, larger deformations would be required in the matrix in order to reach the critical failure stress and the crack is expected to run further along the interface before it eventually kinks in to the matrix at an angle closer to the experiments.

\section{Acknowledgements}

BNL was supported by the Danish Center for Composite Structures and Materials for Wind turbine (DCCSM), supported by The Danish Council for Strategic Research (Grant No. 09-067212).

QDY also wishes to acknowledge the support from from US Army Research Office (ARO Grant No. W911NF-13-10211).

\section{References}

[1] Li, Y., Zheng, Y., Lin, Z., Hu, Z., and Zeng, J., 2012. "Quantitative analysis of inclusions in aluminum". Advanced Materials Research, Adv. Mater. Res, 476-478, pp. 453-456.

[2] McDanels, D., 1985. "Analysis of stress-strain, fracture, and ductility behavior of aluminum matrix composites containing discontinuous silicon carbide reinforcement". Metallurgical Transactions A (Physical Metallurgy and Materials Science), 16A(6), pp. 11051115.

[3] Canal, L. P., Segurado, J., and Llorca, J., 2009. "Failure surface of epoxy-modified fiber-reinforced composites under transverse tension and out-of-plane shear". International Journal of Solids and Structures, 46(11-12), pp. 2265-2274. 
[4] Totry, E., González, C., and Llorca, J., 2008. "Prediction of the failure locus of C/PEEK composites under transverse compression and longitudinal shear through computational micromechanics". Composites Science and Technology, 68(15-16), pp. 3128-3136.

[5] Basu, S., Waas, A. M., and Ambur, D. R., 2006. "Compressive failure of fiber composites under multi-axial loading". Journal of the Mechanics and Physics of Solids, 54(3), pp. 611-634.

[6] Gamstedt, E. K., and Sjögren, B. A., 1999. "Micromechanisms in tension-compression fatigue of composite laminates containing transverse plies". Composites Science and Technology, 59(2), pp. 167-178.

[7] Vajari, D. A., González, C., Llorca, J., and Legarth, B. N., 2014. "A numerical study of the influence of microvoids in the transverse mechanical response of unidirectional composites". Composites Science and Technology, 97, pp. 46-54.

[8] Puck, A., and Schürmann, H., 1998. "Failure analysis of FRP laminates by means of physically based phenomenological models". Composites Science and Technology, 58(7), pp. 1045-1067.

[9] Legarth, B. N., and Kuroda, M., 2004. "Particle debonding using different yield criteria". European Journal of Mechanics - A/Solids, 23(5), pp. 737-751.

[10] Curtin, W. A., 1991. "Theory of mechanical properties of ceramic-matrix composites". Journal of the American Ceramic Society, 45, pp. 2837-2845.

[11] Tvergaard, V., and Legarth, B. N., 2007. "Effects of anisotropic plasticity on mixed mode interface crack growth". Engineering Fracture Mechanics, 74, pp. 2603-2614.

[12] Ling, D., Yang, Q., and Cox, B., 2009. “An augmented finite element method for modeling arbitrary discontinuities in composite materials". International Journal of Fracture, 156, pp. 53-173.

[13] Belytschko, T., and Black, T., 1999. "Elastic crack growth in finite elements with minimal remeshing". International Journal for Numerical Methods in Engineering, 45(5), pp. 601-620.

[14] Zhang, Z. J., Paulino, G. H., and Celes, W., 2007. "Extrinsic cohesive modelling of dynamic fracture and microbranching instability in brittle materials". International Journal for Numerical Methods in Engineering, 72(8), pp. 893-923.

[15] Legarth, B. N., 2005. "Effects of geometrical anisotropy on failure in a plastically anisotropic metal". Engineering Fracture Mechanics, 72(18), pp. 27922807.

[16] Yang, Q., and Thouless, M., 2001. "Mixed-mode fracture analyses of plastically-deforming adhesive joints". International Journal of Fracture, 110(2), pp. 175-187.

[17] Wang, J., and Suo, Z., 1990. "Experimentaldetermination of interfacial toughness curves using brazil-nut-sandwiches". Acta Metallurgica et Materialia, 38(7), pp. 1279-1290.

[18] Fang, X. J., Zhou, Z. Q., Cox, B. N., and Yang, Q. D., 2011. "High-fidelity simulations of multiple fracture processes in a laminated composite in tension". Journal of the Mechanics and Physics of Solids, 59(7), pp. 1355-1373.

[19] Ling, D. S., Fang, X. J., Cox, B. N., and Yang, Q. D., 2011. "Nonlinear fracture analysis of delamination crack jumps in laminated composites". Journal of Aerospace Engineering, 24(2), pp. 181-188.

[20] Moës, N., and Belytschko, T., 2002. "Extended finite element method for cohesive crack growth". Engineering Fracture Mechanics, 69(7), pp. 813-833.

[21] Legarth, B. N., 2004. "Unit cell debonding analyses for arbitrary orientations of plastic anisotropy". International Journal of Solids and Structures, 41(26), pp. 7267-7285.

[22] Paris, F., Correa, E., and Mantič, V., 2007. "Kinking of transversal interface cracks between fiber and matrix". Journal of Applied Mechanics-Transactions of the ASME, 74(4), pp. 703-716.

[23] Ashouri Vajari, D., 2015. "A micromechanical study of porous composites under longitudinal shear and transverse normal loading". Composite Structures, 125, pp. 266-276.

[24] Tvergaard, V., and Hutchinson, J., 1992. "The relation between crack-growth resistance and fracture process parameters in elastic plastic solids". Journal of the Mechanics and Physics of Solids, 40(6), pp. 1377-1397.

[25] Park, S. J., Lee, B. K., Na, M. H., and Kim, D. S., 2013. "Melt-spun shaped fibers with enhanced surface effects: Fiber fabrication, characterization and application to woven scaffolds". Acta Biomaterialia, Acta Biomater, Acta Biomat, Acta Biomater, 9(8), pp. 77197726.

[26] Edie, D. D., Fox, N. K., Barnett, B. C., and Fain, C. C., 1986. "Melt-spun non-circular carbon fibers". Carbon, 24(4), pp. 477-482.

[27] Yeom, B. Y., and Pourdeyhimi, B., 2011. "Web fabrication and characterization of unique winged shaped, area-enhanced fibers via a bicomponent spunbond process". Journal of Materials Science, 46(10), pp. 32523257.

[28] Wang, Y., Liu, X., Wanglei, Lan, X., Xue, J., Jiang, Y., and Zhong, W., 2010. "Preparation and properties of non-circular cross-section sic fibers from a preceramic polymer". Ceramic Transactions, Ceram. Trans, 213, pp. 121-126.

[29] Needleman, A., 1987. "A continuum model for void nucleation by inclusion debonding". Transactions of the ASME. Journal of Applied Mechanics, 54(3), pp. 525-531.

[30] Tvergaard, V., and Hutchinson, J., 1993. "The influence of plasticity on mixed-mode interface toughness". Journal of the Mechanics and Physics of Solids, 41(6), pp. 1119-1135.

[31] Sou, Z., Shih, C., and Varias, A., 1993. "A theory for cleavage cracking in the presence of plastic-flow". Acta Metallurgica et Materialia, 41(5), pp. 1551-1557. 